\title{
Mesure de la hauteur des arbres avec la perche Sokkisha : Test de précision
}

\author{
par Richard Zarnovican ${ }^{1}$ et Jean De Grâce ${ }^{2}$
}

Un test de précision a été effectué avec la perche télescopique Sokkisha pour mesurer la hauteur totale des arbres en comparaison avec la mesure au ruban. Le test a été effectué dans de jeunes sapinières éclaircies de la Haute-Côte-Nord (Québec). Les résultats montrent un faible biais négatif ne dépassant pas $-0,06 \mathrm{~m}$ ou $-1,03 \%$ mais statistiquement significatif au seuil de 0,05 . L'étude de la précision indique une erreur quadratique moyenne ne dépassant pas $\pm 0,11$ $\mathrm{m}$ ou $\pm 1,77 \%$. L'erreur d'une mesure à la perche se situera donc dans un intervalle estimé à $\pm 0,21 \mathrm{~m}$ ou $\pm 3,49 \%$ au niveau de confiance de $95 \%$. Enfin, on est sûr à $95 \%$ qu'au moins $95 \%$ des erreurs de mesures futures de la hauteur à la perche seront situées dans l'intervalle de $\pm 0,23 \mathrm{~m}$ ou $\pm 3,9 \%$.

Mots clés : test de précision, mesure des hauteurs, perche télescopique, dendrométrie, sapin baumier
The accuracy of measuring tree height using a Sokkisha pole compared with that obtained using a tape measure was tested. This test was conducted in young thinned balsam fir stands in the Upper North Shore region of the province of Quebec. The results show a slightly negative bias, $-0.06 \mathrm{~m}$ or $-1.03 \%$, significantly different from zero at the $95 \%$ probability level. A study of the precision shows that the mean quadratic error does not exceed \pm 0.11 $\mathrm{m}$ or $\pm 1.77 \%$. Using the prediction interval, we can be $95 \%$ confident that in a single future measurement, the error will be around $\pm 0.21 \mathrm{~m}$ or $\pm 3.49 \%$. According to tolerance interval, we can be $95 \%$ confident that at least $95 \%$ of the population of errors produced by using the pole will fall between $\pm 0.23 \mathrm{~m}$ or $\pm 3.9 \%$.

Key words: Test of accuracy, measuring poles, tree height measurement, mensuration, balsam fir.

\section{Introduction}

Dans l'inventaire forestier, la hauteur est, après la grosseur de l'arbre, une autre mesure importante pour déterminer le volume ou pour caractériser la qualité des sites. Comme pour les diamètres, on peut définir plusieurs hauteurs. Cependant, dans le cadre de notre étude, nous allons nous limiter à la hauteur totale seulement.

La «hauteur totale» d'un arbre correspond à la distance qui sépare le niveau du sol du bourgeon terminal (Pardé et Bouchon 1988). Cette mesure exprime la hauteur des arbres sur pied. Par ailleurs, la même grandeur mesurée sur un arbre abattu est également désignée comme une longueur totale.

Pour mesurer la hauteur des arbres, on utilise, en plus des dendromètres traditionnels, différentes perches télescopiques (Garthwaite 1981; Holte 1981; Howe et Adams 1988), et ce aussi bien dans le domaine de la recherche que dans la pratique forestière. L'usage de ces instruments est cependant limité aux jeunes arbres dont la hauteur ne dépasse pas $15 \mathrm{~m}$. À l'exception des informations fournies par les fabricants, il existe peu de données sur la précision de ces appareils en foresterie (Holte 1981; Howe et Adams 1988). Cette situation a motivé le présent essai du produit Sokkisha, modèle PSK202. L'étude vise donc à évaluer la précision de cette perche télescopique pour la mesure de la hauteur des arbres sur pied par rapport aux mesures au ruban de la longueur des arbres abattus.

\section{Description et fonctionnement}

Le lecteur pourra trouver une description de la perche et de son mode de fonctionnement dans les différents catalogues de

${ }^{1}$ Ressources naturelles Canada, Service canadien des forêts —Québec, C.P. 3800, Sainte-Foy (Québec), Canada G1V 4C7.

${ }^{2}$ Ressources naturelles Québec, Secteur forêts, UG - Des Escoumins.

fournisseurs d'équipement forestier. Plusieurs modèles sont actuellement offerts sur le marché; ils sont généralement fabriqués en fibre de verre et leur extension maximale varie entre 8 et $15 \mathrm{~m}$. Selon le fabricant, la seule information disponible sur la précision linéaire de ces instruments est de 1/2000 ou de $0,05 \%$. Enfin, selon les données des catalogues, les prix varient entre 400 \$ et 500 \$ (CAN) selon l'élongation maximale de l'instrument.

Une perche est composée de sections qui s'emboîtent l'une dans l'autre. La stabilité d'une perche est assurée par le verrouillage des sections à l'aide d'une rondelle de friction. Pour prendre une mesure, on déploie la perche à proximité de l'arbre et on fait coïncider la tête de la perche avec le bourgeon terminal. La mesure de la hauteur est lue au centimètre près directement sur le ruban déroulé dans le boîtier de la section de base.

\section{Prise de mesures sur le terrain}

L'étude de la précision de la perche Sokkisha, modèle PSK202, a été réalisée dans de jeunes sapinières de la HauteCôte-Nord (Québec), plus précisément aux Escoumins et à SacréCoeur. Les mesures de la hauteur des arbres proviennent de peuplements de 26 ans éclaircis en 1984-1985 dont les densités variaient entre 2000 et 4000 tiges ha $^{-1}$. Les peuplements font partie des régions écologiques $5 \mathrm{~F}$ (sapinière à bouleau jaune, étage supérieur) et $8 \mathrm{E}$ (sapinière à bouleau blanc, étage inférieur) (Thibault 1985). Ils sont situés sur des tills minces et bien drainés. Selon les caractéristiques morphométriques des arbres (tableau 1), les conditions des peuplements sont semblables pour les deux séries de mesures. La hauteur des arbres a été mesurée à la perche et au ruban par des équipes de deux personnes et la prise des mesures a été réalisée en deux étapes à l'automne 1993. Dans un premier temps, deux équipes ont numéroté les arbres sur pied et mesuré leur hauteur à l'aide de 
Tableau 1. Données morphométriques des arbres d'étude

\begin{tabular}{|c|c|c|c|c|}
\hline Variable & Moyenne & Écart-type & Minimum & Maximum \\
\hline \multicolumn{5}{|l|}{ Série -1 (117 arbres) } \\
\hline $\mathrm{Dhp}_{\mathrm{ace}}(\mathrm{cm})$ & 8,6 & 2,2 & 5,6 & 14,2 \\
\hline Hauteur-ruban (m) & 5,78 & 1,16 & 3,20 & 8,11 \\
\hline Hauteur-perche (m) & 5,83 & 1,17 & 3,21 & 8,11 \\
\hline Longueur de cime ${ }^{1}(\mathrm{~m})$ & 4,1 & 1,0 & 2,0 & 6,4 \\
\hline Largeur de cime (m) & 2,0 & 0,4 & 1,3 & 3,0 \\
\hline \multicolumn{5}{|l|}{ Série - 2 (106 arbres) } \\
\hline $\mathrm{Dhp}_{\mathrm{g}}(\mathrm{cm})$ & 8,7 & 2,2 & 5,5 & 14,5 \\
\hline Hauteur-ruban (m) & 6,26 & 1,13 & 3,84 & 8,14 \\
\hline Hauteur-perche (m) & 6,32 & 1,12 & 3,93 & 8,16 \\
\hline Longueur de cime (m) & 4,9 & 1,0 & 3,0 & 6,9 \\
\hline Largeur de cime (m) & 2,1 & 0,4 & 1,3 & 3,2 \\
\hline
\end{tabular}

${ }^{\mathrm{I}}$ Cime verte.

aé = avec écorce.

perches PSK202 lors d'un inventaire forestier réalisé en septembre 1993. Une personne manipulait la perche et lisait la hauteur alors que l'autre personne vérifiait que la tête de la perche coïncidait bien avec le bourgeon terminal de l'arbre et notait la mesure. Les perches ont été calibrées régulièrement avant la prise de mesure.

Dans un deuxième temps, en novembre 1993, deux autres équipes ont mesuré au ruban la longueur de ces mêmes arbres abattus et pris également d'autres mesures morphologiques de l'arbre pour l'analyse de tiges.

La précision de la perche par rapport au ruban est donc évaluée à partir de deux séries de mesures. La première série correspond aux 117 couples de mesures à la perche et au ruban de 88 sapins baumiers (Abies balsamea L. [Mill.]), de 10 épinettes blanches (Picea glauca [Moench] Voss.) et de 19 épinettes noires (Picea mariana [Mill.] B.S.P.). La deuxième série de mesures correspond aux 106 couples de hauteurs, dont 86 sapins baumiers, 11 épinettes blanches et 9 épinettes noires. Au total, la hauteur de 223 arbres a été mesurée à la perche et au ruban.

\section{Sources d'erreurs}

La mesure de la hauteur à la perche télescopique comporte deux types d'erreurs. Le premier type d'erreurs correspond à une erreur instrumentale, reliée au fonctionnement de la perche, alors que le deuxième type correspond aux erreurs aléatoires. Selon l'expérience des équipes sur le terrain, l'erreur instrumentale peut être causée par des sections mal assemblées ou mal verrouillées. On peut les éliminer par l'étalonnage de la perche à l'aide d'un ruban.

Dans le cas des erreurs de mesure ou aléatoires, il est possible d'en évoquer trois principales : 1) la différence entre la base de la perche et la base de l'arbre; 2) l'absence de coïncidence entre la tête de la perche et le bourgeon terminal de l'arbre; et enfin, 3) l'absence de parallélisme entre le tronc de l'arbre et la perche.

\section{Analyse de la précision et objectifs}

Pour évaluer les qualités intrinsèques de la perche télescopique, il est approprié d'évaluer son biais et sa précision, afin de fournir aux forestiers les informations les plus utiles. Dans le cadre de la présente étude nous considérons que les mesures prises au ruban sur des arbres abattus sont sans erreur et que la différence entre la hauteur mesurée au ruban $\left(h_{\mathrm{r}}\right)$ et la hauteur mesurée à la perche $\left(h_{\mathrm{p}}\right)$ est une erreur de mesure $\left(e=h_{\mathrm{r}^{-}}\right.$ $h_{\mathrm{p}}$ ). Notre objectif est donc d'évaluer :

1) la relation entre l'erreur de mesure et la hauteur de l'arbre, en utilisant la régression linéaire simple;

2) l'homogénéité de la variance des erreurs dans le domaine des hauteurs observées, en utilisant le test de Goldfeld-Quandt (Judge et al. 1988);

3) la normalité de la distribution des erreurs par le test de Lillifers;

4) la signification du biais de la perche par le test de Student;

5) la précision de la perche à l'aide des intervalles de prédiction et de tolérance (Reynolds 1984; Rauscher 1986; Rauscher et Harding 1993).

Dans le cas du test de Goldfeld-Quandt, après avoir fait le tri croissant selon la hauteur, les observations ont été divisées en trois groupes. Pour le premier et le troisième groupe d'observations, les sommes des carrés des résidus ont été calculées et le rapport suivant a été établi : $F_{\mathrm{c}}=S_{2} / S_{1}$. La valeur de $F_{\mathrm{c}}$ a été comparée avec la distribution de $F_{\mathrm{a}}$ pour $[(T-r-2 K) / 2$, $(T-r-2 K) / 2$ ] degrés de liberté. Dans cette expression, $T$ est le nombre total d'observations, $r$ est le nombre d'observations non

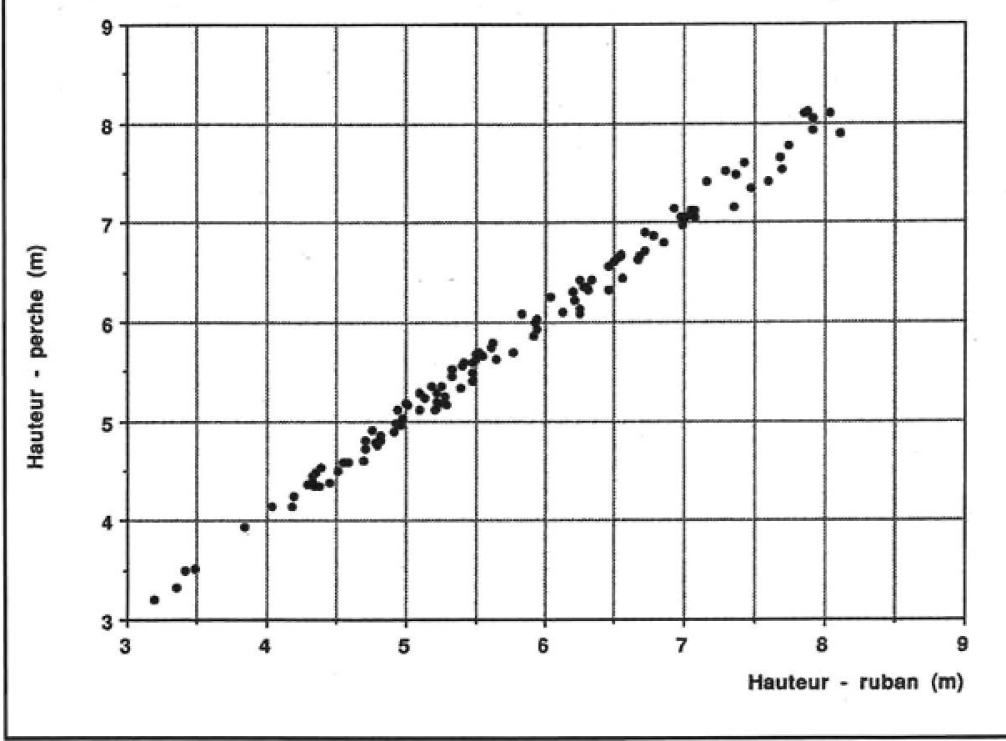

Figure 1. Relation entre la hauteur de l'arbre mesurée à la perche et au ruban - série 1 . 
Figure 2. Relation entre la hauteur de l'arbre mesurée

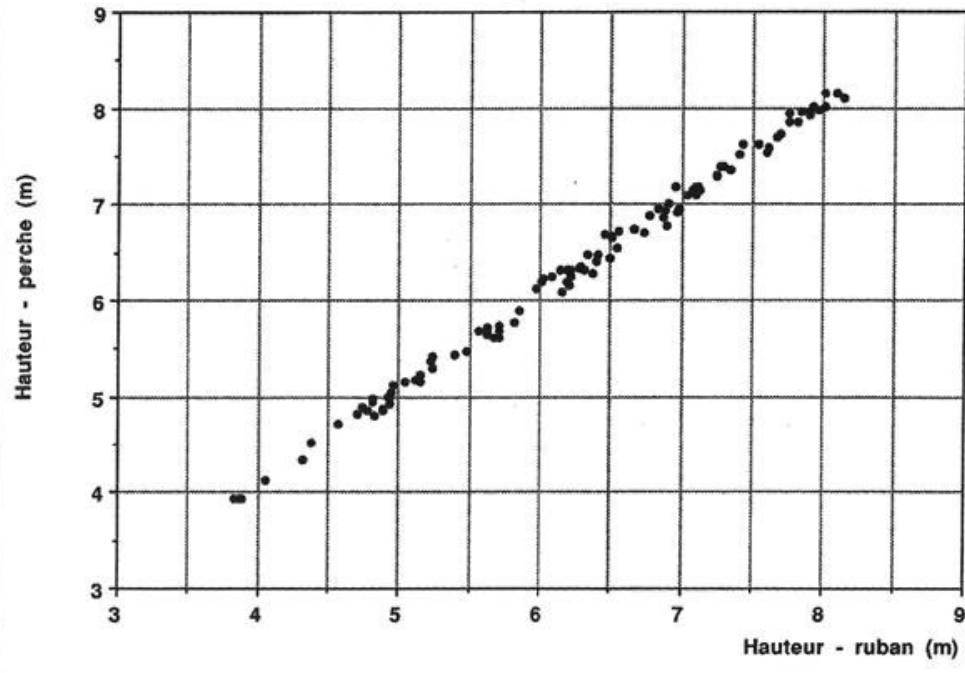

à la perche et au ruban - série 2 .

utilisées et $K$ est le nombre des variables explicatives du modèle.

Pour évaluer la précision de la perche, nous avons établi l'intervalle de prédiction et l'intervalle de tolérance. Dans le cas de l'intervalle de prédiction $\left(I_{\mathrm{p}}\right)$, il estime les limites (inférieures et supérieures) contenant $95 \%$ des erreurs. On a donc une probabilité de 0,95 que la prochaine erreur de mesure se retrouve entre les deux bornes définies par :

$$
\mathrm{Ip}= \pm\left(\frac{1}{\mathrm{n}}+\frac{1}{\mathrm{k}}\right)^{0,5} \sigma \mathrm{t}_{1-\alpha / 2}(\mathrm{n}-1)
$$

où $k=1, n$ est le nombre d'observations, $\sigma$ est l'écart-type des erreurs et $t_{1-\alpha / 2}$ est le (1- $\left.\alpha / 2\right) e$ percentile de la distribution de Student avec $n-1$ degrés de liberté. Enfin, pour établir l'intervalle qui contiendra au moins $95 \%$ d'erreurs des prochaines mesures avec une certitude de $95 \%$, nous avons calculé l'intervalle de tolérance $\left(I_{\mathrm{t}}\right)$ :

$$
\mathrm{I}_{\mathrm{t}}= \pm \mathrm{K}_{(\gamma, \mathrm{n}, \mathrm{p})} \sigma
$$

où $K$ est le facteur de tolérance avec $\gamma$ et $p$ égaux à 0,95 (Eisenhart et al. 1947 dans Rauscher 1986), $\sigma$ est l'écart-type des erreurs et $\mathrm{n}$ est le nombre total d'observations.

\section{Résultats et discussion}

La relation entre la hauteur de la perche et la hauteur au ruban (figures 1 et 2) est très étroite et sans valeurs aberrantes pour les deux séries de mesures. La comparaison de ces deux techniques de mesure (figures 3 et 4 , tableau 2 ) indique qu'entre les hauteurs au ruban et les hauteurs à la perche, il existe des différences négatives systématiques. La moyenne de ces différences, ou le biais, est de $-0,05 \mathrm{~m}(-0,84 \%)$ pour la première série de mesures et de $-0,06 \mathrm{~m}(-1,03 \%)$ pour la deuxième série.

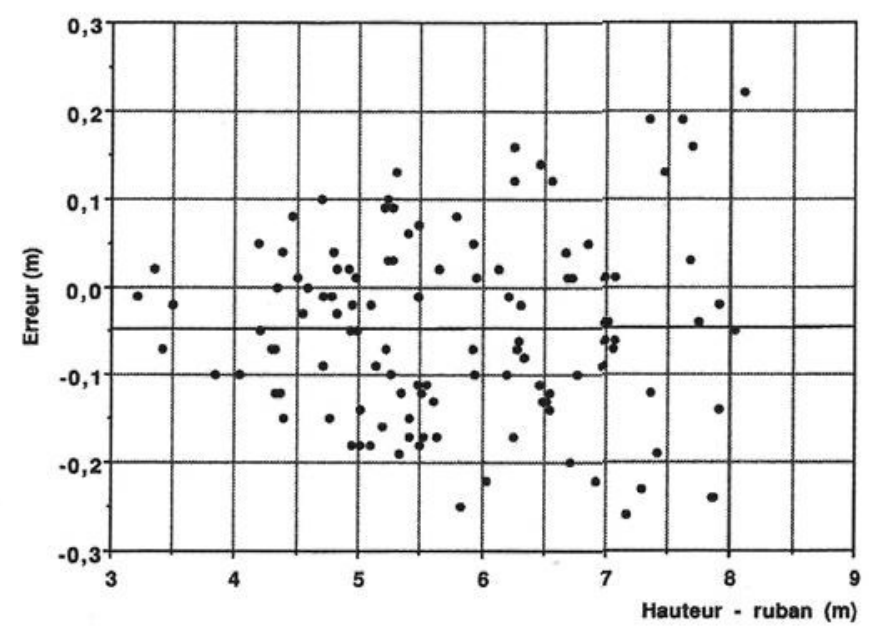

Figure 3. Relation entre l'erreur de la perche et la hauteur mesurée au ruban - série 1. 
Figure 4. Relation entre l'erreur de la perche et la hauteur mesurée au ruban - série 2 .

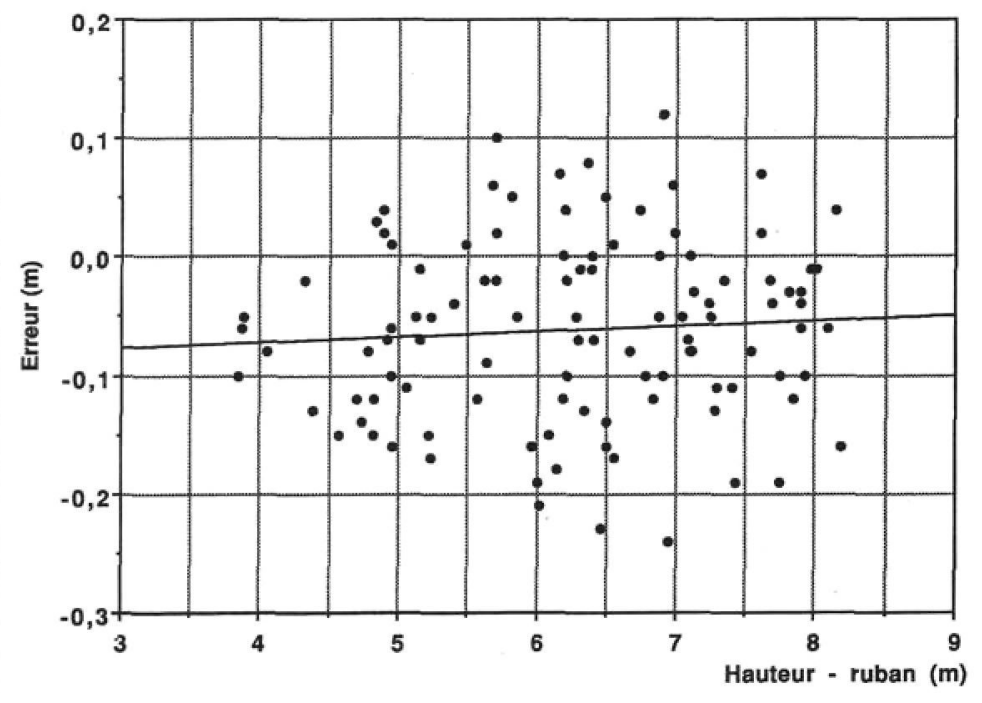

D'après le même tableau, l'écart-type et l'étendue des erreurs sont très proches pour les deux séries de mesures. Selon la régression linéaire simple entre l'erreur de la perche et la hauteur du ruban (figures 3 et 4 ), la liaison entre les deux est pratiquement nulle $\left(\mathrm{R}^{2}\right.$ est de 0,000 pour la première série et de 0,004 pour la deuxième).

L'élargissement des nuages de points en fonction de la hauteur (figures 3 et 4) suggérait l'inégalité des variances dans la distribution d'erreurs. Pour la première série de mesures (figure 3 ), le test de Goldfeld-Quandt $\left(F_{\mathrm{cl}}=3,32\right)$ conclut à l'hétéroscédasticité alors que, pour la deuxième série (figure 4 ), ce même test $\left(F_{\mathrm{c} 2}=0,78\right)$ conclut à l'homoscédasticité, au seuil de $0,05\left(F_{(0,05 ; 34 ; 3)}=1,78\right)$. Dans le cas des erreurs relatives, le même test $\left(F_{\mathrm{c} 1}=1,27 ; F_{\mathrm{c} 2}=1,69\right)$ conclut à l'égalité des variances dans leurs distributions.

Enfin, lorsqu'on vérifie l'hypothèse de normalité pour les deux séries d'erreurs, le test bilatéral de Lillifers (SYSTAT 1992) conclut que la distribution des deux séries ne differe pas de façon significative de la distribution normale et ceci autant pour les erreurs absolues ( $p=0,369$ pour la série 1 et $p=0,641$ pour la série 2), que pour les erreurs relatives ( $p=0,204$ pour la série 1 et $p=0,233$ pour la série 2).

\section{Biais}

Les deux séries de mesures ont été soumises au test de Student, afin de vérifier la signification du biais. On conclut que les biais des deux séries sont différents de zéro de façon significative. La présence de biais significatifs est confirmée par les intervalles de confiance au seuil de $0,05:(-0,07 ;-0,03)$ pour la première série de mesures et $(-0,08 ;-0,05)$ pour la deuxième série de mesures. Ces biais sont pratiquement constants pour les hauteurs mesurées (figures 3 et 4 ).

Le biais négatif des deux séries de mesures indique une surestimation systématique de la hauteur par la perche. Celle-ci résulte sans doute de la différence entre la base de la perche et la base de l'arbre ainsi que de l'absence du parallélisme entre l'arbre et la perche. Parmi les causes possibles, on peut évoquer le microrelief, la présence de la couche organique et également la présence de cimes basses et larges. La présence du biais négatif dans la mesure de la hauteur par la perche a également été constatée par Howe et Adams (1988), lors de la comparaison de la perche avec le clinomètre. Selon ces auteurs, la hauteur estimée avec un clinomètre fut systématiquement plus faible que la hauteur mesurée à la perche. La valeur moyenne de cette différence fut de $0,31 \mathrm{~m}$ pour une hauteur moyenne de $8 \mathrm{~m}$, pour des plantations de sapins Douglas de 15 ans.

\section{Précision}

Les résultats de la présente étude suggèrent que la perche se compare très bien avec différents dendromètres (tableau 3) (Hunt 1959; Bouchon 1967). Cependant, selon les résultats du test de Goldfeld-Quandt, il faut nuancer la précision de la perche lorsqu'on l'exprime en termes absolus ou en termes relatifs. Par exemple, l'imprécision de la perche augmente en fonction de la hauteur des arbres, de $\pm 0,08 \mathrm{~m}$ pour une hauteur moyenne de $4,8 \mathrm{~m}$ à $\pm 0,12 \mathrm{~m}$ pour une hauteur moyenne de $6,7 \mathrm{~m}$ pour la première série de mesures et de $\pm 0,07 \mathrm{~m}$ pour une hauteur moyenne de $5,0 \mathrm{~m}$ à $\pm 0,08 \mathrm{~m}$ pour une hauteur moyenne de $7 \mathrm{~m}$ pour la deuxième série. D'un autre côté, l'erreur relative reste pratiquement constante, de $\pm 1,8 \%$ à $\pm 1,8 \%$ pour la première série de mesures et de $\pm 1,4 \%$ à $\pm 1,2 \%$ pour la deuxième série, pour les mêmes hauteurs moyennes. L'intervalle de prédiction indique que l'erreur de la prochaine mesure à la perche aura une probabilité de $95 \%$ de se retrouver entre $\pm 0,21 \mathrm{~m}$ ou $\pm 3,49 \%$. À long terme, pour un nombre élevé de mesures, on peut être sûr à $95 \%$ qu'au moins $95 \%$ des erreurs à la perche seront situées dans l'intervalle de tolérance, soit $\pm 0,23 \mathrm{~m}$ ou $\pm 3,90 \%$.

Considérant l'imprécision de la perche, on peut se demander si, à toute fin pratique, la lecture de la hauteur au centimètre est réaliste dans les conditions de jeunes peuplements résineux.

\begin{tabular}{lcccr}
\hline $\begin{array}{l}\text { Tableau 2. Statistiques sur les erreurs de la perche } \\
\text { Série } \mathbf{1}\end{array}$ & \multicolumn{2}{c}{ Série $\mathbf{2}$} \\
\cline { 2 - 5 } Erreur & $(\mathrm{m})$ & $(\%)$ & $(\mathrm{m})$ & $(\%)$ \\
\hline Moyenne & $-0,05$ & $-0,84$ & $-0,06$ & $-1,03$ \\
Ecart-type & 0,11 & 1,77 & 0,08 & 1,28 \\
Maximum & 0,22 & 2,71 & 0,12 & 1,75 \\
Minimum & $-0,26$ & $-4,29$ & $-0,24$ & $-3,56$ \\
\hline
\end{tabular}


Tableau 3. Précision de la mesure de hauteur par la perche (pour le seuil bilatéral de 0,05 )

\begin{tabular}{|c|c|c|c|c|}
\hline & \multirow[t]{2}{*}{ Biais } & \multicolumn{2}{|c|}{ Intervalle } & \multirow[t]{2}{*}{ Écart-type } \\
\hline & & de prédiction & de tolérance & \\
\hline $\begin{array}{l}\text { Série I } \\
(\mathrm{m}) \\
(\%)\end{array}$ & $\begin{array}{l}-0,05 \\
-0,84\end{array}$ & $\begin{array}{l} \pm 0,21 \\
\pm 3,49\end{array}$ & $\begin{array}{l} \pm 0,23 \\
\pm 3,90\end{array}$ & $\begin{array}{l}0,11 \\
1,77\end{array}$ \\
\hline $\begin{array}{l}\text { Série } 2 \\
(\mathrm{~m}) \\
(\%)\end{array}$ & $\begin{array}{l}-0,06 \\
-1,03\end{array}$ & $\begin{array}{l} \pm 0,15 \\
\pm 2,54\end{array}$ & $\begin{array}{l} \pm 0,17 \\
\pm 2,85\end{array}$ & $\begin{array}{l}0,08 \\
1,28\end{array}$ \\
\hline
\end{tabular}

Selon l'expérience des équipes, la mesure de la hauteur à la perche au décimètre près peut être tout aussi appropriée. En effet, il $s$ 'avère difficile de contrôler certaines conditions de terrain et de peuplement des jeunes forêts résineuses telles que l'épaisseur d'humus et la grosseur des cimes, lesquelles ont une influence sur la prise de mesures.

\section{Temps de mesure}

Une analyse sommaire du temps de mesure a été effectuée. Elle reflète les conditions d'inventaire par parcelle de 0,1 ha où la hauteur d'un arbre sur quatre a été mesurée dans de jeunes peuplements résineux éclaircis (de 2000 à 4000 tiges à l'hectare). Les résultats indiquent qu'une équipe de deux personnes peut mesurer la hauteur de 35 à 45 arbres à l'heure. Selon l'expérience acquise sur le terrain, la mesure à la perche est plus rapide qu'avec des dendromètres dans les peuplements éclaircis, et ce grâce à la facilité de déplacement et au fait que la perche peut rester constamment déployée.

Cependant, dans les jeunes peuplements denses, le temps de mesure à la perche peut être aussi long qu'au clinomètre, sinon plus. Howe et Adams (1988) ont constaté que le temps de mesure à la perche était de $40 \%$ plus long que le temps de mesure au clinomètre pour une équipe de deux personnes dans un jeune peuplement dense de sapins Douglas.

\section{Conclusion}

La présente étude sur la précision de la perche Sokkisha à mesurer la hauteur totale a été réalisée dans de jeunes sapinières éclaircies de la Haute-Côte-Nord. L'étude a permis d'établir, pour ces conditions de peuplement, que les mesures des hauteurs à la perche ont un biais négatif de $-0,05 \mathrm{~m}(-0,84 \%)$ et de $-0,06 \mathrm{~m}(-1,03 \%)$. Bien que statistiquement significatif, le biais reste constant pour les hauteurs mesurées. La présence du biais a pour conséquence une surestimation systématique de la hauteur, ce qui, selon l'expérience des équipes, pourrait provenir des conditions du terrain et du peuplement. Pour diminuer ou éliminer le biais, il faudra assurer la cö̈ncidence de la perche à la base de l'arbre et au bourgeon terminal ainsi que le parallélisme entre la perche et l'arbre.

La précision de la perche, exprimée par l'erreur moyenne quadratique, est de $\pm 0,11$ m ou de $\pm 1,77 \%$, ce qui en fait un instrument de mesure tout à fait approprié pour l'inventaire forestier. L'intervalle de prédiction pour les erreurs des mesures à la perche est donc de $\pm 0,21 \mathrm{~m}$ ou $\pm 3,49 \%$ avec une probabilité de $95 \%$. Enfin, l'intervalle de tolérance pour au moins $95 \%$ des erreurs est de $\pm 0,234 \mathrm{~m}( \pm 3,9 \%)$ avec une certitude de $95 \%$.

Finalement, selon l'analyse sommaire du temps, on peut s'attendre à un rendement de 35 à 45 arbres à l'heure par une équipe de deux personnes, dans les jeunes peuplements résineux éclaircis.

\section{Bibliographie}

Bouchon, J. 1967. Comparaison de cinq dendromètres pour les mesures de hauteurs d'arbres. Rev. For. Fr. 19: 256-278.

Garthwaite, D.K. 1981. Improved tree height measuring poles. For. Chron. 57: 226-228.

Holte, A. 1981. The accuracy of tree height measurements using measuring poles. Medd. Nor. Inst. Skogforsk 36(2): 1-21.

Howe, G.T. and W.T. Adams. 1988. Clinometer versus pole measurement of tree heights in young Douglas-fir progeny tests. West. J. Appl. For. 3(3): 86-88.

Hunt, E.V., Jr. 1959. A time and accuracy test of some hypsometers. J. For. 57: 641-643.

Judge, G.G., R.C. Hill, W.E. Griffiths and T.C. Lee. 1988. Introduction to the theory and practice of econometrics. John Wiley \& Sons, New York, NY. 1024 p.

Pardé, J. et J. Bouchon. 1988. Dendrométrie. École nationale du génie rural, des eaux et des forêts, Laboratoire d'économie forestière, Nancy, France. 328 p.

Rauscher, H.M. 1986. The microcomputer scientific software series 4: Testing prediction accuracy. US Dept. Agric., North Central Forest Experiment Station, Saint-Paul, Minn., Gen. Tech. Rep. NC107. 19 p.

Rauscher, H.M. and R.B. Harding. 1993. Testing the accuracy of white spruce total-tree volume equations. North. J. Appl. For. 10: 112-116. Reynolds, M.R. 1984. Estimating the error in model predictions. For. Sci. 30: 454-469.

SYSTAT, 1992. Statistics, Version 5.2 Edition. Systat Inc., Evanston, IL. 724 p.

Thibault, M. 1985. Les régions écologiques du Québec méridional (deuxième approximation). Carte synthèse. Ministère de l'Énergie et des Ressources du Québec, Service de recherche et Service de cartographie, Québec, PQ. 